\title{
Prosody in read aloud text: relation with information status, content type and boundary strength
}

Hanny den Ouden ${ }^{1}$ and Carel van Wijk ${ }^{2}$

${ }^{1}$ Faculty of Humanities, UiL/OTS, Utrecht University, The Netherlands

${ }^{2}$ Faculty of Humanities, Tilburg University, The Netherlands

https://doi.org/10.36505/ExLing-2008/02/0020/000079

\begin{abstract}
In two short stories three text characteristics have been implemented independent of each other: information status, content type, and boundary strength. Thus, each characteristic could be assessed for its unique effect on prosody. Both stories were read aloud by ten speakers. No effects were found for pitch range and articulation rate, but pause duration did vary systematically with each of the text characteristics. Effects ranged from small for information status to moderate for content type, and large for boundary strength.
\end{abstract}

Key words: narrative, text characteristics, prosody, pause duration

\section{Introduction}

Research on text prosody has focused on various prosodic features of sentences in relation with their position and function in a text (see e.g. den Ouden, Noordman and Terken, 2008). It has not settled the issue, however, whether effects are real or spurious, that is, does a specific text characteristic exert an influence on its own, or because it happens to coincide with another characteristic actually being the effective one? In this paper we report a study in which, independent of each other, three text characteristics were assessed for their effect on prosody.

\section{Text characteristics}

Three text characteristics have been shown to affect prosody: information structure (van Donzel, 1999), message content (Swerts, Krahmer, Theune and Weegels, 2002), and text organization (den Ouden, 2004). Although some use these terms interchangeably, they represent different perspec-tives on a text as we show here with the texts read aloud in the study.

Information structure is signaled by referential continuity. Does a sentence give information on a referent mentioned in the sentence directly preceding or on some other referent? Box 1 shows how successive sentences switch between both possibilities, denoted [same] and [other].

Message content is defined as the global meaning or gist of a paragraph. Stories are constructed from four basic content types (see Box 2). They start with some background information (state) that leads to a challenging situation (outcome). This takes story characters into a sequence of episodes

ExLing 2008: Proceedings of 2nd Tutorial and Research Workshop on Experimental Linguistics, 25-27 August 2008, Athens, Greece 
in which they want things (goal), try to realize them (attempt), and experience that they have either failed or succeeded (outcome).

Box 1. Illustration of referential continuity (either [same] or [other]).

..... (11) Recently, a businessman had a promising contact with a Columbian (12) He [same] could buy pure cocaine at a low price (13) The police [other] wanted to take advantage of this contact (14) They [same] decided to place a trap for the mafia (15) They [same] wanted to strike drugs traffic very hard (16) Crime in the street [other] had to be pushed back (17) The police [other] formed a team of detectives (18) That [same] had a number of officers infiltrate .......

Box 2. Illustration of content types (for each instance the serial number and content of the central sentence, and serial numbers of surrounding sentences).

\begin{tabular}{|l|l|l|l|}
\hline State & $(6)$ & Circle Island has a shortage of water & $1-10$ \\
\hline outcome & $(11)$ & $\begin{array}{l}\text { Recently, a scientist discovered a cheap method of } \\
\text { water treatment }\end{array}$ & $11-12$ \\
\hline goal & $(14)$ & $\begin{array}{l}\text { The farmers decided to build a canal straight } \\
\text { across the island }\end{array}$ & $13-16$ \\
\hline attempt & $(18)$ & They lobbied a few senators to join & $17-21$ \\
\hline outcome & $(23)$ & The senate, however, voted against & $22-24$ \\
\hline goal & $(26)$ & A smaller canal had to be build & $25-27$ \\
\hline attempt & $(28)$ & The construction of the smaller canal started & $28-30$ \\
\hline outcome & $(33)$ & The canal project had failed & $31-34$ \\
\hline
\end{tabular}

Text organization is scored as boundary strength: the distance between adjacent sentences in terms of the hierarchical structure of the text like the one depicted in Figure 1. Boundary strength is absent for short distances (e.g., from 9 to 10), weak and moderate for longer distances (e.g., from 4 to 5 and 12 to 13), and strong for long distances (e.g., from 16 to 17; for details on scoring, see den Ouden, 2004, p.25).

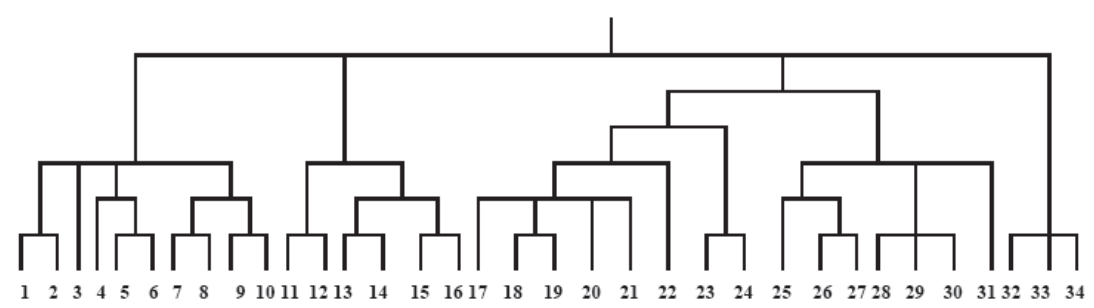

Figure 1. Structure underlying the experimental texts. 


\section{Research questions}

Does each of the three text characteristics affect prosody independent of the other two? And if so, how much do they differ in their effect sizes?

\section{Method}

\section{Material}

Experimental texts were Thorndyke's (1977) Island classic on a political issue and a newly written story on drug crime. Both texts were 34 sentences long. They differed in their contents, but shared the hierarchical structure presented in Figure 1. Each sentence was scored for Information Status (it started with a referent mentioned in directly preceding sentence, or it did not), Content Type (it was part of a paragraph informing on a state, goal, attempt, or outcome), and Boundary strength (its distance from the directly preceding sentence in the hierarchical structure was scored as either absent, weak, moderate, or strong). Within a story each of the text characteristics was distributed sufficiently even, both in number and position. They occurred independent of each other with one exception: strong boundaries tended to coincide less often with a 'same' referent. This trend was too weak to be of any influence on the results.

\section{Procedure}

Ten speakers participated ( 5 men, 5 women). They prepared both texts and then read them aloud (with a third structurally different text read aloud in between the two). Three prosodic features were measured of each sentence: duration of preceding pause, pitch peak and articulation rate. For each prosodic feature repeated measures anova's were run with Gender and Speaker as nested within-factors, with Story and one of the three text characteristics as between-factors, and with the other two text characteristics as covariates. Effect size was determined with partial eta-squared ( $\eta 5)$.

\section{Results}

Pitch peak and articulation rate showed no significant main effects, but pause duration did: for Information Status $(F(1,60)=3.27, \mathrm{p}=.07$, i.e., tested one-sided $\left.\mathrm{p}<.05, \eta^{2}=.05\right)$, Content Type $\left(F(3,56)=3.06, \mathrm{p}<.05, \eta^{2}=.14\right)$, and Boundary Strength $\left(F(3,56)=10.13, \mathrm{p}<.001, \eta^{2}=.35\right)$.Table 1 presents mean pause durations for the three text characteristics. All effects occurred independent of Gender and Story; there were no significant interactions. The effects on pause duration differed in size: small for Information Status, medium for Content Type, and large for Boundary Strength. To illustrate the magnitude of the differences Table 1 also presents the difference of each score from the overall mean pause duration $(718 \mathrm{msec})$. 
Table 1. Pause duration in relation with text characteristics (first line: score in absolute figures, second line: score relative to overall average of 718 msec).

\begin{tabular}{|l|l|l|l|l|l|l|l|l|l|}
\hline $\begin{array}{l}\text { Information } \\
\text { Status }\end{array}$ & \multicolumn{4}{l|}{ ContentType } & \multicolumn{3}{l|}{ Boundary Strength } \\
\hline same & other & state & outcome & goal & attempt & absent & weak & moderate & strong \\
\hline 685 & 746 & 653 & 694 & 767 & 775 & 660 & 696 & 738 & 948 \\
-33 & +28 & -65 & -24 & +49 & +57 & -58 & -22 & +20 & +230 \\
\hline
\end{tabular}

\section{Discussion}

When carefully controlled for confounding effects in the design both of the experimental texts and the statistical analyses, each of the three text characteristics, that is, information status, content type and boundary strength, appears to have a discernable effect on pause duration independent of the other ones. Linguistic theorizing on text prosody and computational generation of artificial speech will have to consider these three text characteristics simultaneously to gain a full understanding of human speech and an adequate mimic in text-to-speech systems.

\section{References}

Donzel, M. van 1999. Prosodic aspects of information structure in discourse. Doctoral dissertation, University of Amsterdam, The Netherlands.

Ouden, H. den 2004. Prosodic realizations of text structure. Doctoral disser-tation, Tilburg University, The Netherlands.

Ouden, H. den, Noordman, L. and Terken, J. 2008. Prosodic realizations of global and local structure and rhetorical relations in read aloud news reports. Speech Communication (in press).

Swerts, M., Krahmer, E., Theune, M. and Weegels, M. 2002. The dual of denial: Two uses of disconfirmations in dialogue and their prosodic correlates. Speech Communication 36, 133-145.

Thorndyke, P. 1977. Cognitive structures in comprehension and memory of narrative discourse. Cognitive Psychology 9, 77-110. 